\title{
A Case of a Bitch with Imperforate Hymen and Hydrocolpos
}

\author{
Shigehisa TSUMAGARI ${ }^{1)}$, Kaoru TAKAGI ${ }^{1)}$, Masatoshi TAKEISHI ${ }^{1)}$ and Mushtaq A. MEMON ${ }^{2)}$ \\ ${ }^{1)}$ Laboratory of Theriogenology, Nihon University, Fujisawa, Kanagawa 252-8510, Japan and ${ }^{2)}$ Department of Veterinary Clinical \\ Sciences, Washington State University, Pullman, WA 99164-6610, U.S.A.
}

(Received 26 July 2000/Accepted 5 January 2001)

ABSTRACT. A 38-month-old female Golden retriever was presented with dysuria and dyschezia. It was difficult to visualize the vagina by vaginoscopy due to a cystic polyp on the hymen. The polyp was $2 \times 3 \mathrm{~cm}$ in diameter, round, and pink in color. From clinical and imaging evaluations the original diagnosis was mucometra or pyometra. From endoscopic examination of the vagina an imperforate hymen was finally diagnosed. The ovaries, uterus, and half of the vagina were removed through a median abdominal incision. The vagina contained about 1.5 liters of fluid, but the uterus and ovaries appeared normal. This is a rare case with imperforate hymen and hydrocolpos with a polyp on the hymenal membrane in bitch.

KEY WORDS: bitch, hydrocolpos, imperforate hymen.

J. Vet. Med. Sci. 63(4): 475-477, 2001

Congenital abnormalities of the canine vagina and vulva are classified into six categories, including four vestibulovaginal ring strictures and two strictures in the vestibulovulvar area [12]. The female genital tract is formed from the Mullerian ducts and urogenital sinus [9]. The hymen is formed by fusion of the Mullerian ducts and urogenital sinus, and in domestic animals disappears during the fetal period or after birth [4, 5, 9]. An imperforate hymen causes hydrocolpos, hydrometra and hydorosalpinx (also hemato-, respectively) [10]. A similar accumulation of vaginal fluid is also caused from vaginal atresia and vaginal aplasia [10]. In the bitch and queen, there are a few reports of imperforate hymen [7, 11] and vaginal atresia [2, 7, 13]. We report a Golden retriever bitch with imperforate hymen and hydrocolpos due to a polyp on the hymen, diagnosed by endoscopic examination.

A 38-month-old female Golden retriever weighing $30 \mathrm{~kg}$ was presented to the Animal Medical Center, Nihon University, with dysuria and dyschezia. The dog had been treated successfully for vaginitis and cystic calculus at 3 months and 2 years of age, respectively. During estrus, 3 months prior to presentation, the owner noticed vulvar swelling (a normal estrous sign), but without proestrous bleeding.

Physical examination revealed a normal bitch except for the cystic polyp in the vagina. Urinalysis revealed a $\mathrm{pH} 6$, slight protein and occult blood, 20/hpf red blood cells and 8/ hpf white blood cells consisting mainly of neutrophils were observed in the urine sediment, and the urine specific gravity was 1.030 . The size of the cystic polyp prevented full digital exploration of the cranial vagina, but no vulvar discharge was noted. Left lateral (Fig. 1-A) and ventrodorsal (Fig. 1-B) radiographs of the abdomen revealed an extremely enlarged vagina $(15 \times 27 \mathrm{~cm})$, initially diagnosed as the uterus, located above the urinary bladder and reaching to the posterior margin of the liver. On the ventrodorsal view, the cranial pole of the urinary bladder extended to the last rib, displaced toward the right side of the abdomen, with a very long proximal urethra. The vagina, which was thought to be a uterus simplex, displaced to the left side, occupied two-thirds of the abdomen. About $850 \mathrm{~m} l$ urine was collected at pneumocystography. On ultrasonography, the vaginal canal appeared as a fluid filled cavity and was apparently different from the normal urinary bladder. It was difficult to recognize the urinary bladder and vagina without urethral catheterization (Fig. 2).

At the owner's request, the bitch was treated first with tromethamine dinoprost $\left(\mathrm{PGF}_{2 \alpha}\right)(0.025-0.25 \mathrm{mg} / \mathrm{kg}, \mathrm{SC}$, bid) [6]. No obvious benefit of the treatment was recognized, and $\mathrm{PGF}_{2 \alpha}$ therapy was stopped after 5 days. Serum progesterone decreased from $8.5 \mathrm{ng} / \mathrm{m} l$ before the therapy, to $2.0 \mathrm{ng} / \mathrm{m} l$ and $0.7 \mathrm{ng} / \mathrm{ml}$ on days 2 and 4, respectively.

Endoscopic examination of the vagina revealed an intact hymen beyond the external urethral orifice, and a cystic polyp $(2 \times 3 \mathrm{~cm})$ containing $1 \mathrm{ml}$ of serous fluid on the outside of the mucosal membrane of the hymen (Fig. 3). Imperforate persistent hymen with hydrocolpos was the final diagnosis.

For surgical treatment, the dog was premedicated with atropine sulfate $(0.02 \mathrm{mg} / \mathrm{kg}), \mathrm{SC}$ and midazolam $(0.5 \mathrm{mg} /$ $\mathrm{kg}), \mathrm{IV}$, induced with thiopental sodium $(10 \mathrm{mg} / \mathrm{kg}), \mathrm{IV}$, and maintained with sevoflurane. A urinary catheter was inserted into the urethra (Fig. 3). Ovaries, uterus, and twothirds of the vagina were removed by ovariohysterectomy after median laparotomy. The caudal portion of the vagina was adhered extensively to the proximal urinary bladder and the rectum. An episiotomy was performed and the affected part of the hymen was dissected, following irrigation in the caudal vagina with saline solution. The dissected portion of the hymen was sutured around the vaginal wall and completely opened. About 1.5 liters of brown-red fluid was removed from the vagina (Fig. 4).

No bacteria were isolated on aerobic and anaerobic cultures of the fluid. Degenerative macrophages, neutrophils and red blood cells were observed in the sediment of the fluid. Visual examination of the reproductive tract revealed a small amount of fluid in the uterus; the cervix was closed. 


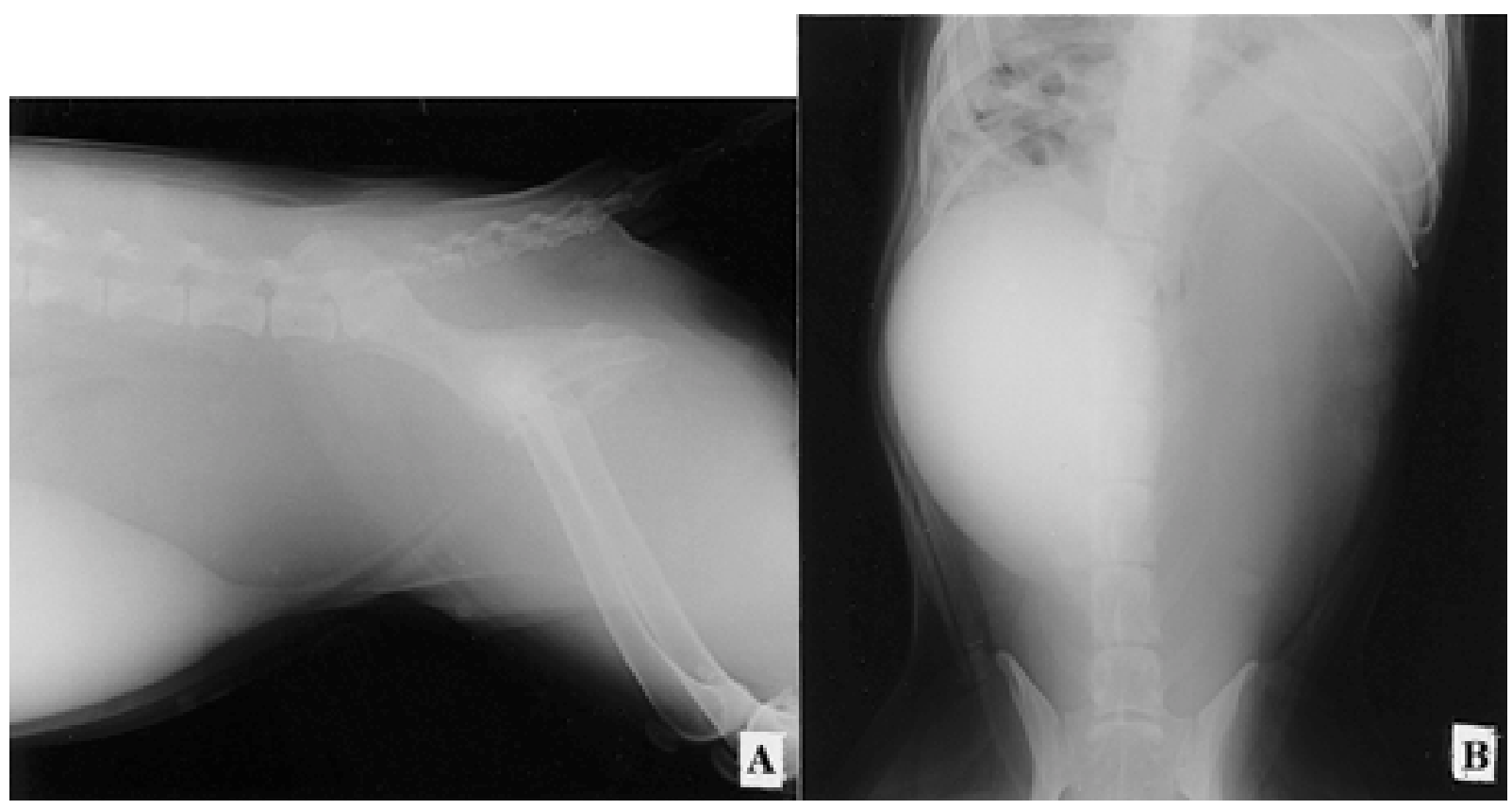

Figs. 1-A and -B. Left lateral (A) and ventrodosal (B) radiographs of the abdomen. There is an apparent difference between the X-ray density in urinary bladder and vagina, in spite of no use of contrast medium.

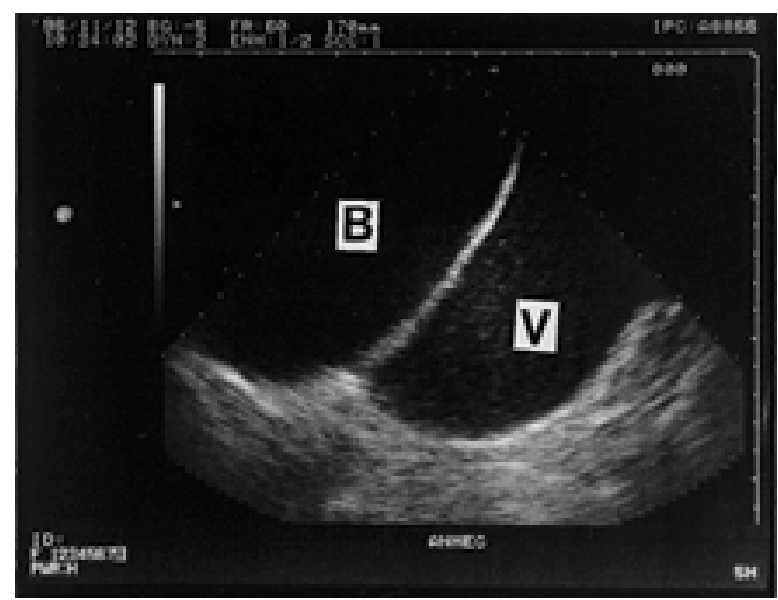

Fig. 2. Transverse sonogram of the vaginal cavity (V) and bladder (B).

Corpora lutea were present on both ovaries (Fig. 5). On histopathologic examination, a few neutrophils, macrophages, and lymphocytes were observed in the submucosa of the vaginal wall near the cervix. Moderate hypertrophy of the endometrium, similar to a diestrual canine uterus, was noted.

Post surgical therapy included amoxicillin $(20 \mathrm{mg} / \mathrm{kg}$, PO, bid) for two weeks. Due to persistent signs of dysuria, the animal urinated through a urinary catheter every day and bladder irrigation was done with $0.2 \%$ povidon-iodine solution every other day, for one week. After one postoperative

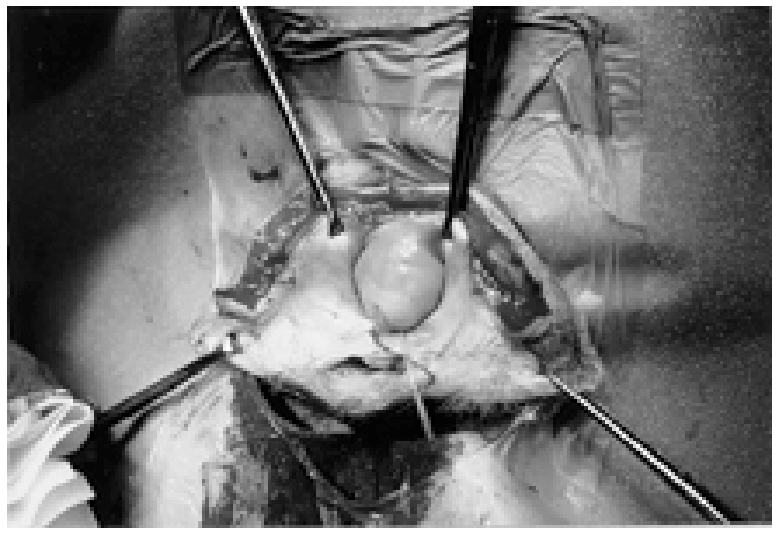

Fig. 3. The cystic polyp $(2 \times 3 \mathrm{~cm})$ on the hymen at the perineal incision.

week, the bitch was given dantrolene sodium $(2 \mathrm{mg} / \mathrm{kg}$, PO, bid) and prazocin hydrochloride $(0.1 \mathrm{mg} / \mathrm{kg}, \mathrm{PO}$, bid) for three weeks to relax the urethra. Spontaneous urination resumed four days after starting treatment and recovered completely two weeks after beginning treatment. She developed incontinence of urine in 20 months postoperatively.

Bitches with defects of the vagina and vulva usually show vulvar discharge, chronic vulvar licking, or attract male dogs [1]. Root et al. [8] described inability to breed naturally, dysuria, ambiguous external genitalia, and dystocia as the primary clinical signs of vaginal stenosis. The subject of 


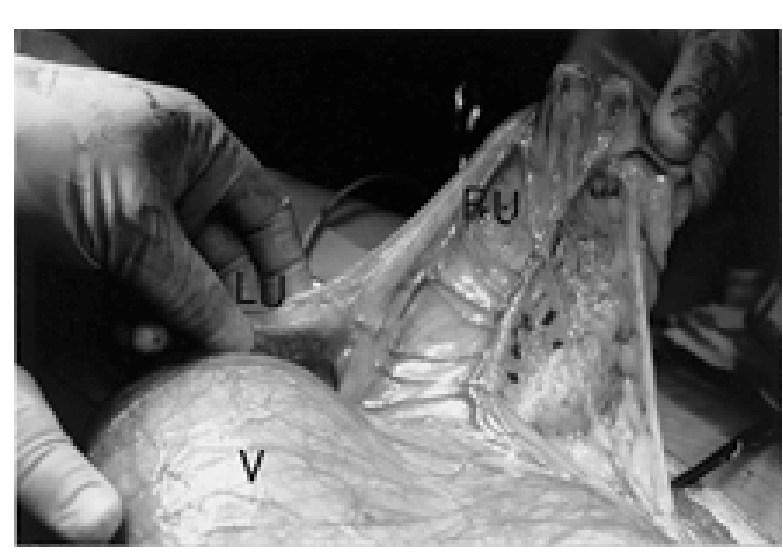

Fig. 4. Laparotomy showed both uteri (RU and LU) and a huge vagina $(\mathrm{V})$.

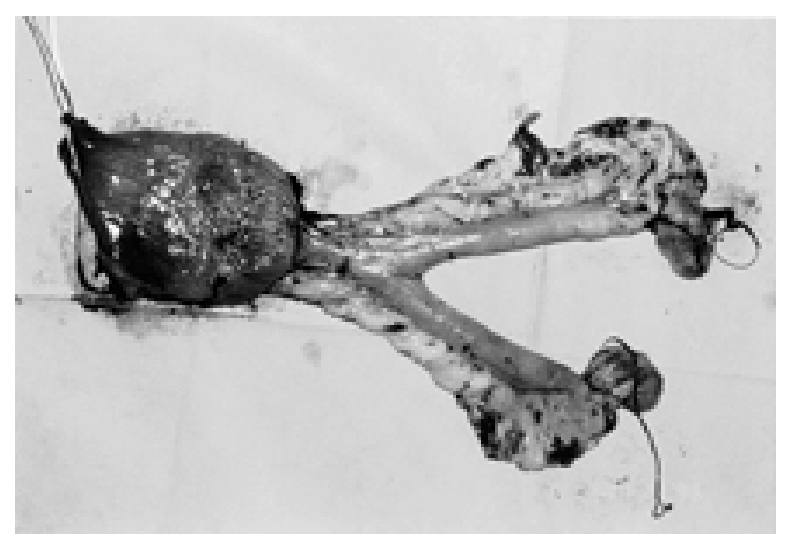

Fig. 5. Ovaries, uterus and part of the vagina shrunk after extirpation.

this report was treated for vaginitis at three months, but the cause was unknown. At presentation, the hymen was not visible by vaginoscopy because of a cystic polyp on the hymenal membrane. Therefore, she was diagnosed as having mucometra or pyometra from the results of other examinations. Endoscopy or positive-contrast vaginography may be necessary to diagnose vaginostenosis in middle or large sized bitches [8]. Almost all cases of hydrocolpos or hematocolpos are founded by exploratory surgery [7, 13].

Imperforate hymen and vaginal atresia involve similar clinical signs or diseases; dysurea, dyschezia, cystitis, and renal failure, etc $[2,7]$. After several estrous periods the accumulated fluid in the vagina may result in displacement of the urinary bladder and expansion of the urethra [7]. The dysuria could have been caused by functional urethral narrowing. For treatment in such a case, muscular relaxation of the urethra might promote spontaneous urination.

Prognosis of hydrocolpos or hematocolpos depends on the degree of renal failure $[2,7,13]$. The subject developed incontinence of urine in 20 months postoperatively, without renal failure.

\section{REFERENCES}

1. Feldman, E.C. and Nelson, R.W. 1996. pp. 648-653. In: Canine and Feline Endocrinology and Reproduction, 2nd ed., W.B. Saunders Co., Philadelphia.

2. Hawe, R.S. and Loeb, A.F. 1984. J. Am. Anim. Hosp. Assoc. 20: $123-130$.

3. Holt, P.E. and Sayle, B. 1981. J. Small Anim. Pract. 22: 67.

4. Jubb, K.V.F. and Kennedy, P.C. 1970. p. 492. In: Pathology of Domestic Animals, 2nd ed., Academic Press, New York.

5. McEntee, K. 1990. p. 198. In: Reproduction Pathology of Domestic Mammals. Academic Press Inc, San Diego.

6. Memon, M.A. and Mickelsen, W.D. 1993. J. Am. Vet. Med. Assoc. 203: 509-511.

7. Nomura, K. and Okuda, K. 1986. J. Jpn. Vet. Med. Assoc. 39: $149-154$.

8. Root, M.V., Johnston, S.D. and Johnston, G.R. 1995. J. Am. Vet. Med. Assoc. 206: 56-58.

9. Sadler, T.W. 1985. pp. 265-271. In: Langman's Medical Embryology, 5th ed. The Williams \& Wilkins Co., Baltimore.

10. Sato, T. 1992. pp. 391-393. In: Clinical Guidelines on Obstetrics and Gynecology. Chugaiigakusya, Tokyo (in Japanese).

11. Smith, K.W. 1965. pp. 644-645. In: Canine Surgery (Archibald, J. ed.), American Veterinary Publications Inc, Illinois.

12. Wykes, P.M. and Soderberg, S.F. 1983. J. Am. Anim. Hosp. Assoc. 19: 995-1000.

13. Yamada, S. 1997. J. Jpn. Vet. Med. Assoc. 50: 603-605. 